\title{
EPTCAST - UNA PROPOSTA PER LA DIVULGAZIONE SCIENTIFICA NELL'EDUCAZIONE PROFESSIONALE E TECNOLOGICA BRASILIANA
}

\section{ARTICOLO ORIGINALE}

RAMOS, Rogério Luiz da Silva', FECURY, Amanda Alves², OLIVEIRA, Euzébio de ${ }^{3}$, DENDASCK, Carla Viana ${ }^{4}$, DIAS, Claudio Alberto Gellis de Mattos $^{5}$

RAMOS, Rogério Luiz da Silva. Et al. EPTcast - Una proposta per la divulgazione scientifica nell'educazione professionale e tecnologica brasiliana. Revista Científica Multidisciplinar Núcleo do Conhecimento. Anno 06, Ed. 06, Vol. 07, pp. 89103. giugno 2021. ISSN: 2448-0959, Link di accesso: https://www.nucleodoconhecimento.com.br/formazione-it/professionale-etecnologica, $\quad$ DOI: $\quad 10.32749 /$ nucleodoconhecimento.com.br/formazioneit/professionale-e-tecnologica

\section{RIEPILOGO}

Questo lavoro consiste in un rapporto di esperienza riguardante lo sviluppo di EPTCast, prodotto didattico sviluppato durante tutto il master professionale in Formazione Professionale e Tecnologica in Rete Nazionale (ProfEPT). Basato sulla mancanza di divulgazione scientifica specificamente focalizzata sul campo dell'educazione professionale e tecnologica brasiliana (EPT), questo lavoro mira a

\footnotetext{
${ }^{1}$ Laureato in Commercio Estero, Specialista in Ingegneria della Produzione (UNINTER), Professore e ricercatore presso I'Istituto di Educazione Di Base, Tecnica e Tecnologica di Amapá (IFAP), Studente del Corso di Laurea in Formazione Professionale e Tecnologica (PROFEPT IFAP).

${ }^{2}$ Biomedicale, Dottorato di Ricerca in Malattie Tropiche, Professore e ricercatore del Corso di Medicina del Campus Macapá, Università Federale di Amapá (UNIFAP).

${ }^{3}$ Biologo, Dottore di Ricerca in Malattie Tropiche, Professore e ricercatore del Corso di Educazione Fisica,Università Federale di Pará (UFPA).

${ }^{4}$ Teologo, PhD in Psicoanalisi Clinica. Si occupa da 15 anni di Metodologia Scientifica (Metodo di Ricerca) nell'Orientamento alla Produzione Scientifica per Master e Dottorandi. Specialista in Ricerche di Mercato e Ricerche Sanitarie Dottorando in Comunicazione e Semiotica (PUC SP).

${ }^{5}$ Biologo, Dottore di Ricerca in Teoria e Comportamento, Professore e ricercatore del Corso di Laurea in Chimica dell'Istituto di Educazione Di Base, Tecnica e Tecnologica di Amapá (IFAP) e del Corso di Laurea in Formazione Professionale e Tecnologica (PROFEPT IFAP).
} 
promuovere iniziative in questo senso, contribuendo così alla divulgazione della scientificità di quest'area. A tal fine, qui viene ritratto il processo di costruzione e distribuzione tramite podcast di un contenuto audio finalizzato alla diffusione scientifica dell'EPT brasiliano. In questo senso, inizialmente viene affrontata la questione del divario e, quindi, la necessità di popolare la scientificità di quest'area. In un secondo momento vengono presentati il design e le tecnologie utilizzate nella costruzione dell'oggetto EPTCast. La popolarità della scientificità dell'educazione professionale e tecnologica brasiliana è ciò che si intende attraverso questo lavoro, che non sarà I'unico. Attraverso ciò che è stato esposto nelle sezioni precedenti, quindi, si intende contribuire alla popolarità di una scientificità che a volte non è ancora intesa come tale.

Parole chiave: Podcast, Divulgazione Scientifica, Formazione Professionale e Tecnologica.

\section{INTRODUZIONE}

La divulgazione scientifica è una pratica adottata dalla premessa della distanza tra la comunità scientifica e il pubblico non specializzato. In questo senso, questo sforzo comunicativo è stato presentato come uno strumento pedagogico discorsivo di costruzione della conoscenza che mira ad avvicinare i processi scientifici al grande pubblico (MOIRAND, et ali,2016).

Pertanto, con l'avvento della diffusione della rete mondiale di computer attraverso Internet, la divulgazione scientifica trova più spazio per svilupparsi. Questo viene percepito attraverso la varietà di media digitali in cui si manifesta questa attività: dai testi su blog, videosu piattaforme dedicate e anche attraverso podcast (DE-LARAGONZÁLEZ e DEL-CAMPO-CAÑIZARES, 2018).

Con il suo primo riferimento datato nel 2004, il podcast multimediale è diventato popolare nel campo della comunicazione. Dalla crescente quantità e varietà di opzioni, la gente comune, le aziende e persino le società di media tradizionali hanno prodotto e distribuito i loro contenuti attraverso questi media (RIVERA SALAS e MONTOYA MÁRQUEZ, 2019). 
Di fronte al già consolidato, il multimediale podcast ha processi semplici e accessibili, sia per la sua produzione che per il suo consumo. Pertanto, questo si presenta come una ragione per utilizzare questi media nella distribuzione di contenuti educativi. Tuttavia, quando si tratta di divulgazione scientifica, questo movimento è meno frequente (DANTAS-QUEIROZ, WENTZEL e QUEIROZ 2018).

In particolare per quanto riguarda la divulgazione scientifica nell'educazione professionale e tecnologica brasiliana (EPT), c'è un vuoto. Inteso come strumento di ascensione sociale, questo settore di ricerca ha costruito le conoscenze necessarie per migliorare le condizioni delle classi lavoratrici popolari in modo che, di fatto, si sviluppi la società nel suo complesso (MOURA, 2013).

\section{OBIETTIVO}

Ritrarre il processo di costruzione di EPTCast al fine di promuovere iniziative più grandi e migliori, al fine di contribuire alla popolarità della scientificità prodotta nel campo dell'EPT brasiliano.

\section{MATERIALI E METODI}

Sviluppato da coloro che vedono l'integrazione tra Istruzione e Lavoro come un percorso per lo sviluppo sociale delle classi lavoratrici popolari, EPTCast è costituito attraverso un contenuto audio finalizzato alla diffusione scientifica dell'EPT brasiliano. Distribuito attraverso il podcast multimediale, questo è un prodotto educativo iniziato nella prima metà del 2020 e sviluppato durante tutto il master professionale in Formazione Professionale e Tecnologica in Rete Nazionale (ProfEPT).

La prima sfida per la costruzione di questa proposta è stata la creazione di un'identità visiva, che richiedeva la progettazione di un nome, uno slogan e un simbolo grafico. Un'identità visiva è, di regola, progettata per la migliore comunicazione possibile attraverso segni verbali e/o non verbali (MILLER e TOMAN, 2014). Pertanto, data la necessità di distribuzione attraverso Internet - che richiede fortemente la presenza di 
risorse visive per la propagazione dei contenuti (LIU, 2020) - questa è stata la prima richiesta nella costruzione di questo Prodotto Educativo.

Quindi, per la concezione del nome, inizialmente è stata data la preferenza per qualcosa di obiettivo e che era direttamente associato al suo modo di comunicare. In questo senso, e soprattutto, dalla consapevolezza che i nomi - sia per idee, prodotti o servizi - sono sempre costruiti all'interno di una struttura esistente, che fa sì che un nuovo nome porti parte di altri (NDERITU PATRICK, 2016), abbiamo scelto di allearsi con l'acronimo di "Educazione Professionale e Tecnologica" con l'ultima parte delnome dei suoi mezzi di distribuzione 'cast'. Di conseguenza, è stato creato il nome EPTCast.

Poi, per la continuazione dello sviluppo dell'identità visiva, è stato costruito uno slogan. Breve frase e rapida associazione con l'idea proposta per nome, uno slogan mira a presentare brevemente il concetto di un'opera (MILLER e TOMAN, 2014). Pertanto, abbiamo scelto di descrivere i supporti di distribuzione insieme al contenuto proposto. Presto, il nome EPTCast ha adottato il seguente slogan: "Il tuo podcast sull'educazione professionale e tecnologica".

Infine, dopo la concezione del nome e del suo slogan,è stato seguito dalla costruzione di un simbolo grafico. Conosciuta anche come logo, questa è una caratteristica visiva che mira alla comunicazione con il pubblico interessato. In questa prospettiva, l'identità visiva di un marchio dovrebbe essere uno strumento di comunicazione semplice e intelligibile (BOLHUIS et ali,2015). Pertanto, abbiamo scelto l'uso di un palloncino di dialogo. Infine, per la costruzione dell'idea elaborata, abbiamo fatto uso della versione gratuita della piattaforma online per la creazione di disegni e layout, Canva.com.

A seguito dello sviluppo dell'identità visiva, la materializzazione del Prodotto Educativo EPTCast ha avuto le attività produttive e distributivi. La produzione consisteva nel lavoro di scripting, acquisizione e modifica di contenuti audio. Per queste attività, il software di editing del testo Microsoft Word versione 2016, il software di editing audio Audacity versione 2.3.1 e le funzionalità audio delle piattaforme YouTube (audioteca) e Freesound.org sono stati utilizzati come strumenti di lavoro. 
A sua volta, la Distribuzione è stata effettuata attraverso l'uso del Anchor.fm. Per l'identificazione del materiale prodotto, gli spazi dedicati a questo compito sono stati utilizzati direttamente sulla piattaforma Anchor.fm, che ha inserito automaticamente i contenuti di EPTCast nelle seguenti piattaforme audio digitali: Apple Podcasts; Demolitore; Castbox; Google Podcasts; Nuvoloso; Calchi tascabili; RadioPublic e Spotify.

\section{RISULTATI E DISCUSSIONI}

Il lavoro utilizzato durante tutto il processo di sviluppo di questo Prodotto Educativo è stato il risultato di ore di consumo precedente di contenuti di divulgazione scientifica distribuiti tramite podcast. Tuttavia, poiché vi sono rari riferimenti nell'area del EPT brasiliano nel caso di queste specificità, i seguenti risultati possono, allo stesso tempo, presentare limitazioni per quanto riguarda la sua costruzione e opportunità per lo sviluppo di proposte più solide.

\section{IDENTITÀ VISIVA}

Attraverso l'utilizzo della versione gratuita della piattaforma Canva.com (https:canva.com) è iniziata la costruzione dell'identità visiva del Prodotto Educativo EPTCast. Per la tela è stata scelta la dimensione di 1500 per 380 pixel e il colore \#000000. Per il nome, il carattere tipografico Montserrat Classic in stile grassetto con la taglia 50 e il colore della \#000000. Quindi, lo slogan è stato lavorato, cheha mantenuto lo stesso carattere tipografico e lo stesso stile applicato al nome, ma ha ricevuto cambiamenti nelle dimensioni e nel colore, rispettivamente 38 e \#ffffff. Infine, è stata selezionata una finestra di dialogo all'interno dell'opzione 'Forme' in 'Elementi' sulla piattaforma in uso. Dopo l'inserimento, l'elemento è stato ridimensionato fino a dimensioni 365 per 290 pixel e il colore \#ffbf00 (Figura 1). 
Figura 1 - Identità visiva del prodotto educativo EPTCast.

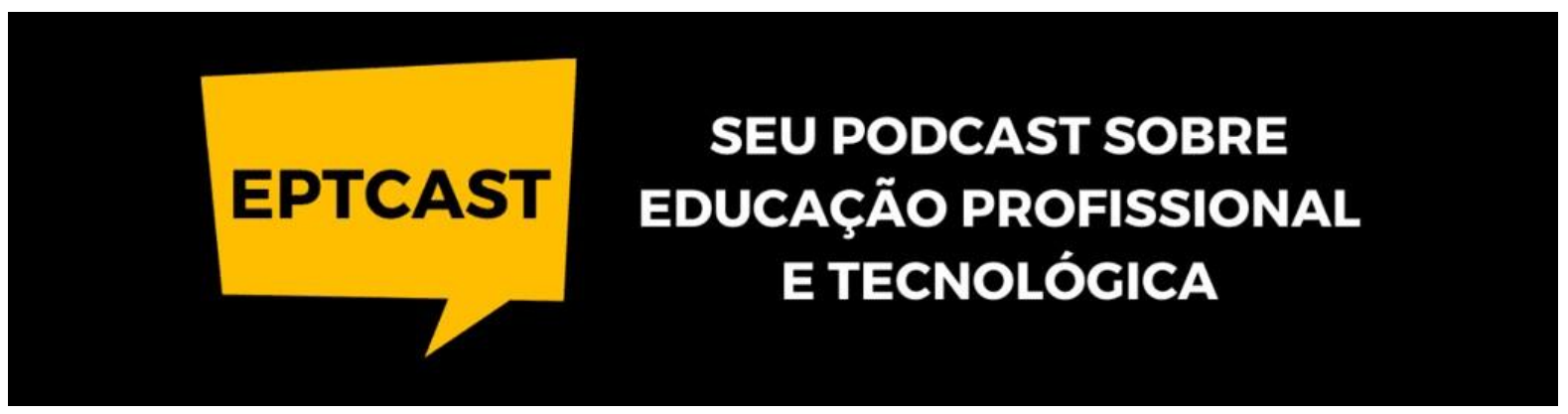

L'identità visiva di questo Prodotto Educativo è stata progettata in modo che potesse essere applicata in futuro negli spazi digitali e nei prodotti promozionali fisici. Pertanto, fin dall'inizio della sua costruzione, è stato stabilito che questa identità dovrebbe essere caratterizzata attraverso una semplice immagine e molteplici applicabilità.

Allinterno di questa proposta, al nome, sono state applicate specifiche che hanno soddisfatto il previsto. Al fine di mantenere la caratteristica centrale del nome, lo slogan ha conservato la stessa idea, tuttavia, dato il maggior numero di lettere, le sue dimensioni sono state ridotte e, per essere letto davanti alla tela, il suo colore è stato cambiato. II Pallone del Dialogo è stato richiesto per il riconoscimento della proposta comunicativa. II suo colore differenziato e la possibilità di posizionare il nome di fronte ad esso è stato progettato in modo che potessero anche essere presentati in modo indipendente allo slogan.

\section{PRODUZIONE}

Da gennaio 2020 a settembre dello stesso anno, la produzione di questo prodotto educativo ha portato a 19 audio (\#00 a \#18). All'inizio - fino alla pubblicazione del 12 il contenuto è stato sviluppato attraverso soggetti ricorrenti nella letteratura del EPT brasiliano. Dal 14 inizia a produrre e pubblicare attraverso suggerimenti di argomenti inviati dal pubblico, che si sono utilizzati dei canali di comunicazione eptcast.com.br (commenti) e della via e-mail contato.eptcast.com.br. II $13^{\circ}$ audio pubblicato aveva lo scopo di riassumere i contenuti finora presentati e stimolare la partecipazione del pubblico. 


\section{SCRIPTING}

Dopo il completamento dell'identità visiva, lo scripting è stato eseguito tramite l'utilizzo del software proprietario di modifica del testo Microsoft Word, versione 2016 (https://office.com). Dall'uso di questo strumento è iniziata la costruzione del contenuto sviluppato in 03 parti, "Presentation", "Evolution" e "Conclusion" (Tabella 1).

La "Presentazione" è stata suddivisa in altre 03 parti, dove, la prima 'Opening', è stata dedicata all'accoglienza, alla presentazione dell'annunciatore e all'identificazione del programma. Poi, 'Context', si è concentrato sull'identificazione della congiuntura del fenomeno affrontato. Terminando il blocco "Presentazione",viene utilizzato il blocco "Call to action",nel momento in cui il pubblico è invitato a interagire per via elettronica alla fine dell'audio.

Un attimo dopo, "Evolution", fu diviso in 04 parti. II primo, 'Phenomenon', è stato dedicato al riassunto dell'oggetto trattato. I successivi "Antecedenti" e "Caratteristiche" sono stati spazi focalizzati sull'approccio del processo di sviluppo del fenomeno in questione e delle sue proprietà. Infine, "Desdobramentos", si è dedicato alle conseguenze nel contesto sociale, in particolare per l'istruzione e il lavoro.

La "Conclusione" è stata divisa in 03 parti. II primo, "Reflection", è stato dedicato a osservazioni concise sull'argomento trattato. Un attimo dopo, 'Riferimenti', mirava a informare il pubblico sul luogo di alloggio del materiale utilizzato nella costruzione della sceneggiatura. Infine, èstata utilizzata la funzione "Invito all'azione". 
Tabella 1 - Schema narrativo della sceneggiatura.

\begin{tabular}{|c|c|c|}
\hline Apresentação & Evolução & Conclusão \\
\hline Abertura & Fenômeno & Reflexão \\
\hline (30 segundos) & (01 min. e 30 segs.) & (02 mins. e 30 segs.) \\
\hline Contexto & Antecedentes & Referências \\
\hline (01 minuto) & (01 min. e 30 segs.) & (30 segundos) \\
\hline \multirow{3}{*}{$\begin{array}{r}\text { Call to action } \\
\text { (30 segundos) }\end{array}$} & Características & Call to action \\
\hline & (03 minutos.) & (01 minuto) \\
\hline & $\begin{array}{c}\text { Desdobramentos } \\
(03 \text { minutos. })\end{array}$ & \\
\hline 02 minutos & 09 minutos & 04 minutos \\
\hline
\end{tabular}

\section{5 minutos}

Gli elementi compositivi dello schema narrativo sono stati pensati in modo tale da far comprendere all'ascoltatore il rapporto tra il fenomeno trattato e la realtà attuale nel contesto dell'Educazione e del Lavoro. II tempo dedicato a ciascuna divisione, e alle sue suddivisioni, era stabilito in modo tale da consentire la standardizzazione, e quindi una migliore organizzazione per la costruzione di itinerari futuri.

Dei 18 audio prodotti, i numeri \#00, \#04, \#08, \#10 e \#12, a causa della natura delle loro proposte, non erano conformi al sistema di cui sopra. E, dal \#13, a causa della pluralità tra le suggestioni dei temi ricevuti, lo schema narrativo ha subito alcuni cambiamenti nella sezione "Evoluzione". Tuttavia, l'essenza di ciò che è stato costruito nello schema presentato sopra, è stata preservata fino a quando l'ultimo soggetto pubblicato

La tabella 2 mostra il numero di audio e di argomenti affrontati. 
Tabella 2 - Temi rivolti a ciascun audio.

\begin{tabular}{c|c}
\hline Número & Assunto \\
\hline$\# 00$ & O que é o EPTCast?"; \\
\hline$\# 01$ & Manual ou Intelectual? \\
\hline$\# 02$ & Onde estão as fontes? \\
\hline$\# 03$ & Natural ou Social? \\
\hline$\# 04$ & O que é... Ciência? \\
\hline$\# 05$ & A crise de 29 \\
\hline$\# 06$ & Globalização \\
\hline$\# 07$ & Matemática \\
\hline$\# 08$ & A Fala Docente! \\
\hline$\# 09$ & O que é Materialismo Histórico Dialético? \\
\hline$\# 10$ & Final de Temporada \\
\hline$\# 11$ & Percurso Histórico Estrutural da EP Brasileira \\
\hline$\# 12$ & A Cientificidade da Música \\
\hline$\# 13$ & Educação Profissional e Propedêutica \\
\hline$\# 14$ & Redação \\
\hline$\# 15$ & Cursos Técnicos \\
\hline$\# 16$ & Formação de Professores para EPT \\
\hline$\# 17$ & Novas Exigências e Velhas Transformações \\
\hline$\# 18$ & \\
\hline
\end{tabular}

Fino a quando I\#16 corsi tecnici, il tempo di ogni produzione è stato di 15 minuti. Da questo, quindi, \#17 nuovi requisiti e vecchie trasformazioni; e \#18 formazione degli insegnanti per EPT, sono stati prodotti e pubblicati con un tempo totale di 12 minuti. Questo cambiamento è stato promosso a causa dei suggerimenti del pubblico. 


\section{CATTURA}

Dopo la conclusione dello schema narrativo e l'elaborazione della prima sceneggiatura, iniziò la produzione audio. A tal fine, l'acquisizione è stata eseguita tramite software di audacia, versione 2.3.1 (https://audacityteam.org) installata su un microcomputer portatile modello Lenovo IdeaPad 310 e con l'ausilio di un microfono a condensatore generico, il BM-800 (Figura 2).

Figura 2 - Acquisizione audio.

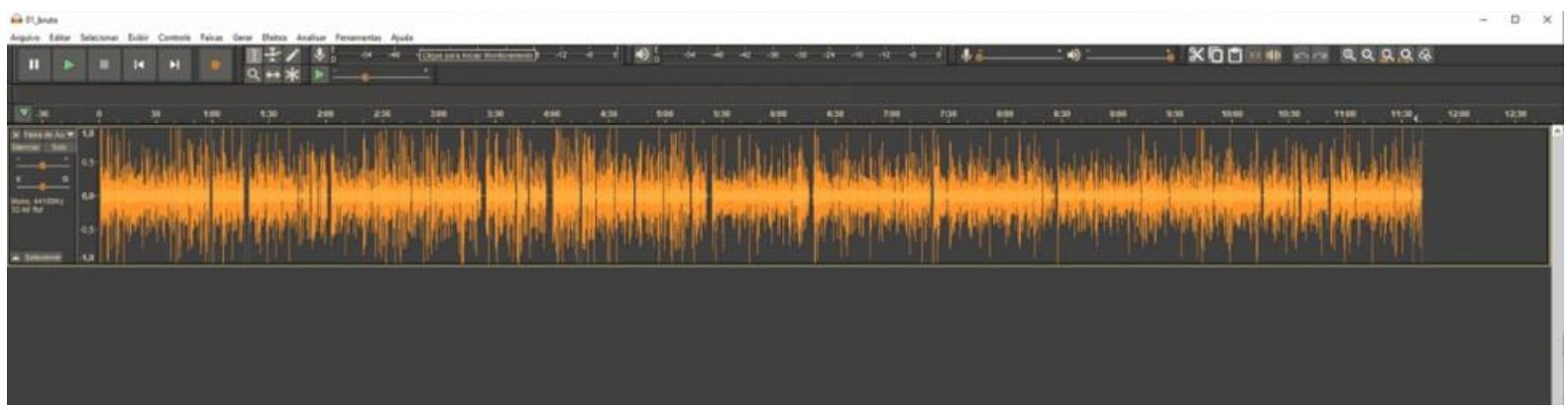

L'acquisizione audio ha rappresentato l'inizio del processo di materializzazione dell'obiettivo pianificato. A causa della mancanza di esperienza in ciò che si riferiva alla narrazione di contenuti scritti per la distribuzione audio, la dimensione / volume della registrazione era a volte più del doppio del tempo stabilito per ogni audio. La figura precedente mostra il contenuto registrato senza gli errori del processo di acquisizione.

\section{EDIZIONE}

Dopo aver acquisito l'audio e rimosso il suo surplus, è stato avviato il processo di modifica. Per questo stadio, le risorse audio delle piattaforme YouTube (https://youtube.com/audiolibrary) sonostate utilizzate per l'inserimento di brani musicali e Freesound.org (https://freesound.org) per l'inserimento di effetti sonori. Entrambe le piattaforme presentano contenuti senza costi finanziari (figura 3). 
Figura 3 - Editing audio.

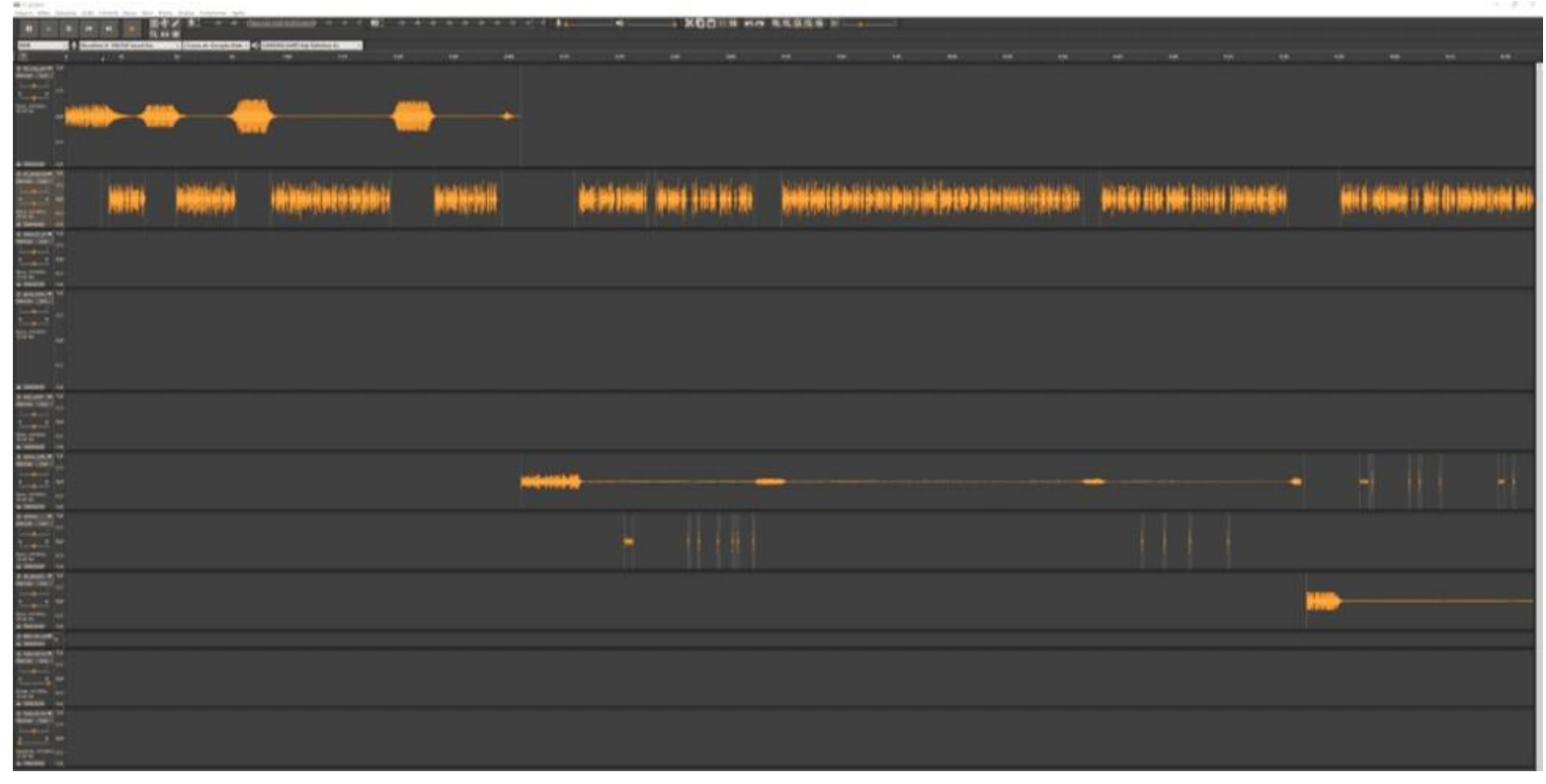

L'opzione di inserire tracce musicali ed effetti sonori è stata il risultato dell'esperienza del consumo precedente di contenuti distribuiti tramite podcast. Durante tutto il processo di editing, è stato anche percepito che questa risorsa, ad un certo livello, si è presentata come in grado di ammorbidire la presenza di alcuni inserimenti indesiderati, provenienti dall'acquisizione audio nell'ambiente domestico.

Al fine di differenziare il discorso del narratore con gli argomenti originali delle fonti di ricerca, il contenuto aveva la partecipazione a rotazione di 04 studenti della Scuola Superiore Integrata dell'Istituto Federale di Istruzione, essendo 02 di Amapá, campus di Santana; e 02 del Rio Grande do Sul, campus di Osório. In questo senso, gli studenti invitati erano responsabili della narrazione delle citazioni dirette inserite nel testo della sceneggiatura.

\section{DISTRIBUIZIONE}

Tra il gran numero di opzioni per la distribuzione di EPTCast, abbiamo optato per il Anchor.fm - http://anchor.fm. Si trattava di una scelta basata su due fondamentali, vale a dire: gratuità totale nella sua manipolazione; distribuzione automatica dei contenuti pubblicati su altre piattaforme. 


\section{IDENTIFICAZIONE DEL MATERIALE}

Inviando il contenuto prodotto attraverso la risorsa dedicata a questa attività (Click to upload or drag files here), l'audioè stato caricato, consentendo così la riproduzione in modo da poter controllare la qualità del contenuto da distribuire (Figura 4).

Figura 4 - Inserimento dell'audio e identificazione del materiale.

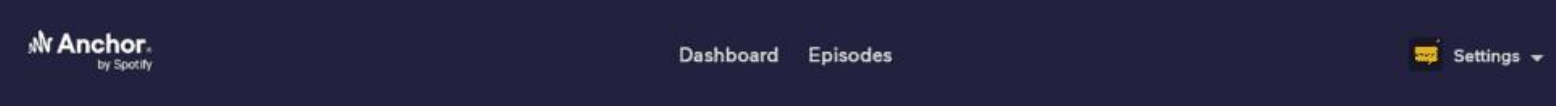

\section{Create your episode}
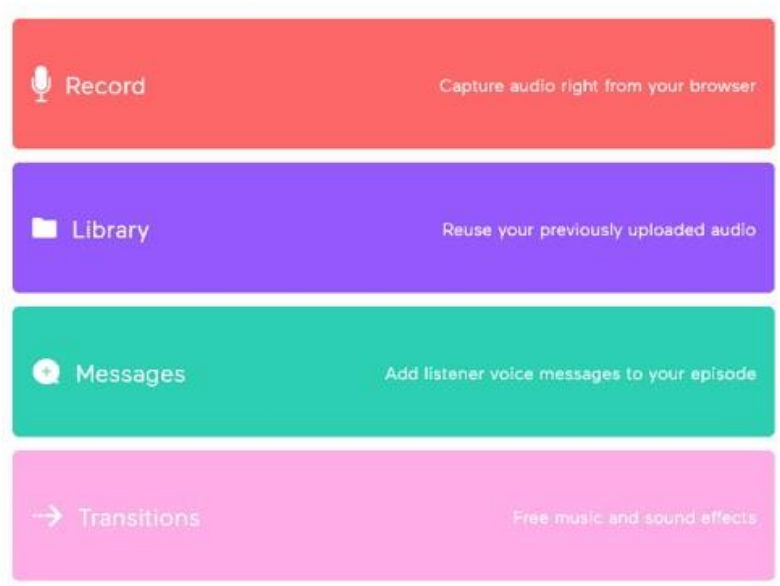

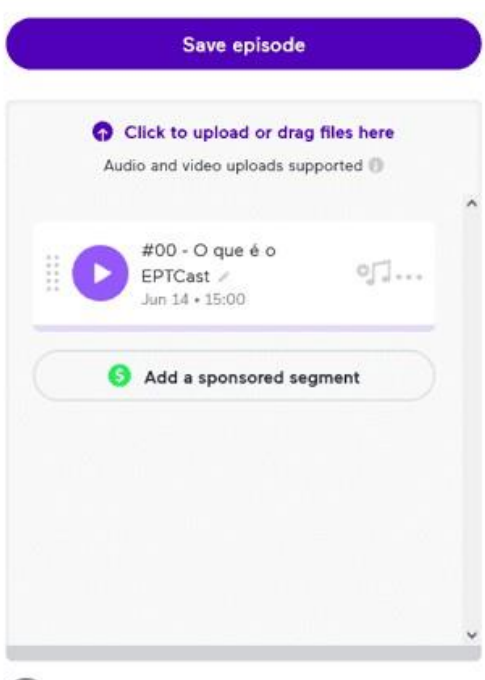

Preview Episode

Dopo aver inserito l'audio, la piattaforma ha richiesto le informazioni di base per identificare il contenuto. Questi erano: titolo (Episode title), descrizione (Episode description), stagione (Season number), numero del contenuto (Episode number), tipo di contenuto tra: completo, trailer o bonus (Full; Trailer; Bonus) e infine, se questo è contenuto user-friendly o meno (Clean; Explicit) (Figura 5). 
Figura 5 - Identificazione del materiale.

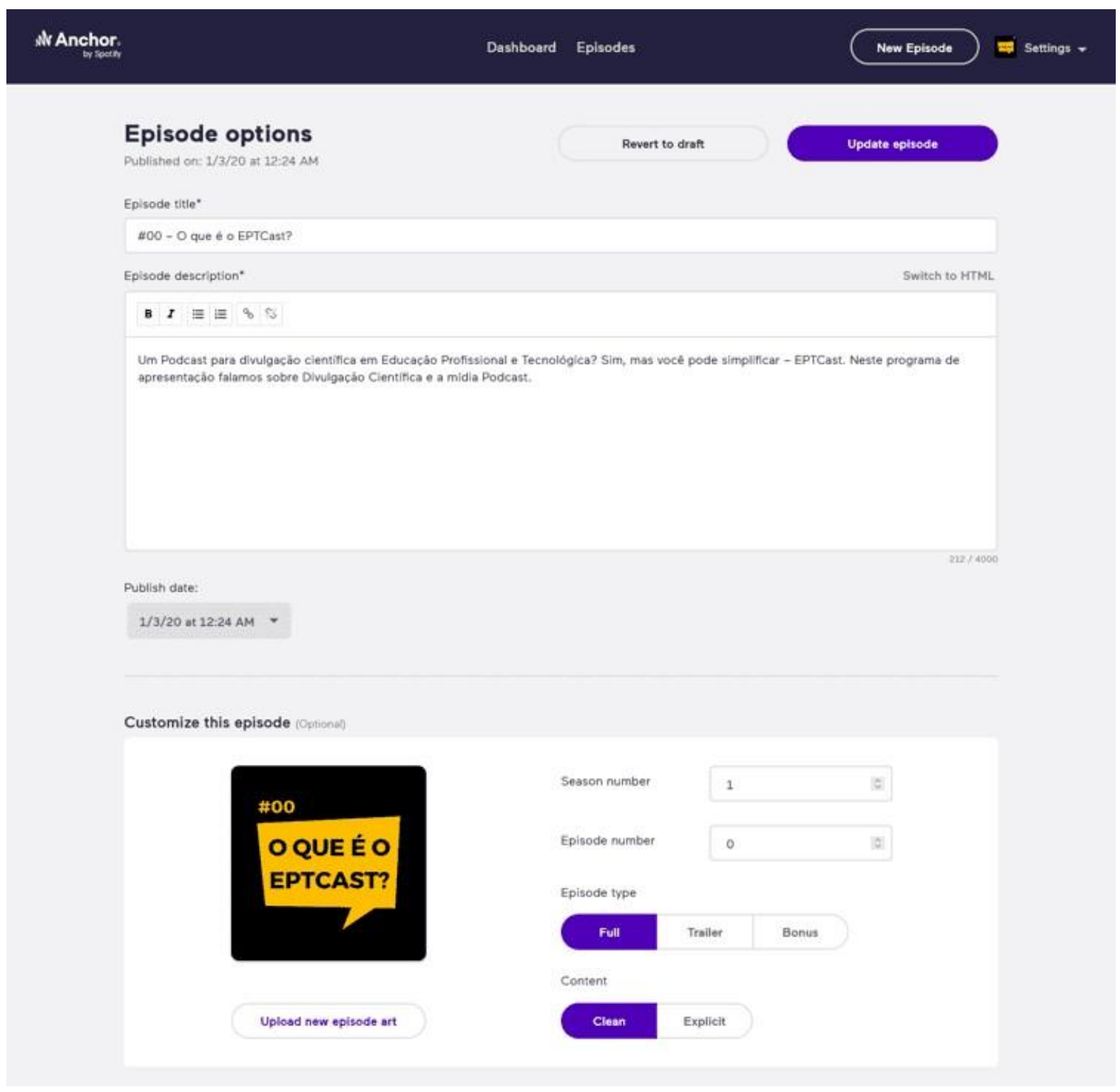

Dopo l'inserimento delle informazioni richieste per l'identificazione dei contenuti, l'audio è stato reso disponibile su questa piattaforma, e anche nella piattaforma di servizi di streaming musicale Spotify (https://spotify.com), consentendone così il consumo nei primi istanti. 


\section{PIATTAFORME AUDIO DIGITALI}

Nonostante l'automazione relativa al processo di distribuzione su altre piattaforme, Anchor.fm non ha eseguito questa attività contemporaneamente su tutte le piattaforme a cui è associato. Tuttavia, poiché l'invio di audio aumentava i contenuti online di EPTCast, si notò che le opzioni di accesso stavano crescendo. Nel distacco del \#07 Globalizzazione, è stato osservato che la distribuzione ha raggiunto il suo numero massimo di piattaforme, 09 (tabella 3).

Tabella 3 - Piattaforme di accesso.

\begin{tabular}{c|c}
\hline NOME & ENDEREÇO (URL) \\
\hline Anchor & anchor.fm/eptcast \\
\hline Breaker & breaker.audio/eptcast \\
\hline Castbox & castbox.fm/channel/id2581365 \\
\hline Google Podcasts & google.com/podcasts?feed=aHROcHM6Ly9hbmNob3luZm0vc \\
\hline Apple Podcasts & podcasts.apple.com/us/podcast/eptcast/id1494934631 \\
\hline Overcast & overcast.fm/itunes1494934631/eptcast \\
\hline Pocket Casts & pca.st/8znyqbx5 \\
\hline RadioPublic & radiopublic.com/eptcast-WkaRNN \\
\hline Spotify & open.spotify.com/show/1hZ3KSkDDzOHuLmIPD6dh8
\end{tabular}

La distribuzione attraverso la piattaforma selezionata si è presentata come un'esperienza di facile comprensione e anche di semplice maneggevolezza. L'inserimento in altre piattaforme digitali, gratuitamente, si è presentato come un importante differenziale poiché questa attività ha dimostrato di far risparmiare tempo, una risorsa applicata nello svolgimento di altri compiti di questo Prodotto Educativo.

\section{CONSIDERAZIONI FINALI}

La popolarità della scientificità dell'educazione professionale e tecnologica brasiliana è ciò che era inteso attraverso questo lavoro, che non sarà l'unico. Attraverso ciò che è stato esposto nelle sezioni precedenti, quindi, si intende contribuire alla popolarità di una scientificità che a volte non è ancora intesa come tale. 
Lo sviluppo sociale delle classi lavoratrici popolari, attraverso lo sviluppo di una cultura scientifica, è la nostra intenzione. In questo senso, ci facciamo ricorso a un media decentralizzato per rendere popolari questi temi che, pur essendo focalizzati sull'emancipazione di coloro che hanno bisogno, finora, si trovano e si udino solo all'interno del ciclo riservato.

Da qui, ci troviamo fiduciosi in iniziative maggiori e migliori volte a popolare questi problemi.

\section{RIFERIMENTI}

MOIRAND, S. et ali. La vulgarisation scientifique au croisement de nouvelles sphères d'activité langagière. Bakhtiniana, Rev. Estud. Discurso, vol. 11 n. 2, p. 137-161, 2016.

DE-LARA-GONZÁLEZ, A.; DEL-CAMPO-CAÑIZARES, E. El podcast como medio de divulgación científica y su capacidad para conectar con la audiencia. Revista Mediterránea de Comunicación, vol. 09 n. 1 p. 347-359, 2018.

RIVERA SALAS, P.E.; MONTOYA MÁRQUEZ, O. R. Evaluación de los podcasts de noticias: un acercamiento a emisoras de la ciudad de puebla. Revista de Comunicación de la SEECI. n. 48, p. 109-123, 2019.

DANTAS-QUEIROZ, M. V.; WENTZEL, L. C. P.; QUEIROZ, L. L. Science communication podcasting in Brazil: the potential and challenges depicted by two podcasts. An. Acad. Bras. Cienc., v. 90, n. 2, p. 1891-1901, 2018.

MOURA, D. H. Ensino médio integrado: subsunção aos interesses do capital ou travessia para a formação humana integral? Educ. Pesqui., v. 39, n. 3, p. 705-720, 2013.

NDERITU PATRICK G. Bilingual naming of business premises, products and services in Murang'a county. Relatório de Projeto de Pesquisa (Mestre em Linguística) Universidade de Nairóbi, 2016. 
LIU, X. Application of the Internet Technology in the Visual Communication Design. In: Atiquzzaman M., Yen N., Xu Z. (eds) BDCPS 2019, AISC 1117, p. 1701-1706, 2020.

MILLER, D. W.; TOMAN, M. An analysis of rhetorical figures and other linguistic devices in corporation brand slogans. Journal of Marketing Communications, v. 22, n. 5, p. 474-493, 2014.

Inviato: Giugno 2021.

Approvato: Giugno 2021. 